\title{
Was bedeutet Wissenschaft und Literatur für eine gelehrte Gesellschaft?
}

\author{
Wissenschafts- und Literaturtheorie in Preßburg \\ im 18. Jahrhundert
}

\section{Einleitung}

Im 18. Jahrhundert, im Jahrhundert der Aufklärung und der Gesellschaften - wie Ulrich im Hof formulierte -, kann man im damaligen Ungarischen Königreich schwer eine besser dokumentierte Gesellschaft, als die Pressburgische Gesellschaft der Freunde der Wissenschaften finden, welche wahrscheinlich unter der Führung des späteren Bürgermeisters und Zeitungsherausgebers, Karl Gottlieb Windisch stand. Wir kennen nahezu die Namen der Mitglieder, die Themen, worüber Vorträge gehalten wurden, und manche Texte auch. Als ich vor siebzehn Jahren über die Archivalien dieser Gesellschaft einen Vortrag gehalten habe, und zwei Jahre später denselben publizierte, ${ }^{1}$ dachte ich nicht, dass ich später zu diesen Forschungen zurückkehren würde. Doch eine bedeutende Frage, die mich als Philologe und Literaturwissenschaftler sehr interessiert, blieb bis heute ungeklärt. Warum nannten sich die Preßburger mit Selbstbewusstsein wissenschaftliche Gesellschaft, wenn sie im heutigen Sinne gar nicht nur über wissenschaftliche Themen diskutierten? Was bedeutet eigentlich Wissenschaft im Kontext der Zeitgenossen, wieso wurde sie von anderen Feldern der Wissensübergabe abgetrennt?

In den letzten Jahren habe ich mich damit beschäftigt, wodurch sich eine Textmenge aus den Litterae, aus der Gesamtheit der geschriebenen gelehrten Werke separiert hat, und gleichzeitig (bis heute geltend) eine neue Interpretationsstrategie annimmt, unabhängig davon, ob man das heute Primär- oder Sekundärliteratur nennt. Meine Annahme ist, dass die Themenwahl der Vorträge und Aufsätze einer gelehrten Gesellschaft eine Antwort auf meine neuerliche Frage geben kann.

\footnotetext{
1 Béla Hegedüs: Über die Pressburgische Gesellschaft der Freunde der Wissenschaften. In: Deutsche Sprache und Kultur im Raum Pressburg. Hg. von Wynfried Kriegleder u.a. Bremen 2002 (Presse und Geschichte - Neue Beiträge 4), S. 53-64.
} 
Im folgenden, besonders wenn es um die möglichst kurze Darstellung der Pressburgischen Gesellschaft und um eine Gesellschaftstypologie geht, werde ich meine vorherigen Forschungsergebnisse mit einbeziehen, da, wie erwähnt, die Ausgangspunkte dieses Aufsatzes in meinen damaligen Ergebnissen fundieren.

\section{Gelehrte Gesellschaften}

Zuerst über die sogenannten gelehrten oder wissenschaftlichen Gesellschaften allgemein. Man kann zwei Typen der wissenschaftlichen Gesellschaften unterscheiden. Es gibt Pläne für akademische Vereinigungen, die man, wenn sie zustande kamen, Elitegesellschaften nennt; sie etablierten sich meistens in den Hof- oder Regierungszentren. Diese kann man im engeren Sinne Gelehrten-, sogar akademische Gesellschaften nennen. Es wird in der neuesten Forschung behauptet, dass diese Gesellschaften in einem früher nicht vorausgesetzten Zusammenhang im Interesse der aktuellen Regierung standen, wie das z. B. Steffen Martus in seiner großen Monographie über die deutsche Aufklärungsepoche erklärt. ${ }^{2}$

Daneben standen die wissenschaftlichen Gesellschaften, deren Mitglieder keine - oder nicht vor allem - namhaften Gelehrten sind, durch ihre Zielsetzung aber, nämlich durch die Selbstbildung und die Förderung kultureller Bestrebungen, an wissenschaftlichen Diskussionen teilnehmen wollen. Auch der Mangel einer wissenschaftlichen Öffentlichkeit gab Anlaß zur Gründung solcher Gesellschaften. Ihr Entstehen beweist das Selbstbewusstsein der Gründer. Die Wissenschaft ist nur ein Mittel für die gemeinnützigen Bestrebungen, was auch die Selbstbildung einschließt. In einer zweiten Stufe der Geschichte der Gesellschaften werden dann die Ergebnisse an das Publikum als publizierte Aufsätze verbreitet. Im Falle der Gesellschaften des ehemaligen Königreichs Ungarn - auch der Pressburgischen - gab es allgemein noch eine weitere Zielsetzung: die Mitglieder wollten durch ihre Arbeit dem Ausland beweisen, dass das kulturelle und wissenschaftliche Leben des vielsprachigen Karpatenbeckens bedeutende Ergebnisse vorweisen konnte. Das sprachlich determinierte Selbstbewusstsein einer Gesellschaft - ihre nationale Identität in modernem Sinne - hat sich in dieser Gegend im Vergleich mit Westeuropa später entwickelt. Die sogenannte Hungarus-

2 Steffen Martus: Aufklärung: Das deutsche 18. Jahrhundert. Ein Epochenbild. Berlin 2015. Das Thema wurde besonders im ersten Teil (Die Anfänge der Aufklärung) im Kapitel „Die Berliner Akademie der Wissenschaften: Hofpolitik und Policeyeinsatz“ ausgeführt. Ebd., S. 82-91. 
Identität der gebildeten Menschen - was nicht bedeutete, Ungar zu sein - hat sich erst am Ende des 18. Jahrhundert aufgelöst. ${ }^{3}$

Die Gesellschaften des 18. Jahrhunderts wollten selten Elitegesellschaften sein. Sie wollten vor allem die Möglichkeit der freien Kommunikation und des freien Diskurses sichern, welche das veränderte, sich von der Autorität voriger Texte und Personen befreite, Wissen nahe legte, und wozu den Hintergrund das soziale und politische Programm der Aufklärung gab. Da aber in Osteuropa dieses Programm gar keine Wirkung ausübte (meiner Meinung nach kann man in unseren Gegenden bis heute nicht behaupten, dass sich die Aufklärung im westeuropäischen Sinne abgespielt hätte), sie war immer eine Sache weniger Intellektueller. Daraus folgt, dass die Freimaurerlogen Osteuropas in der Entwicklung zur heutigen relativ (ich betone: relativ) offenen Gesellschaft vielleicht eine noch wichtigere Bedeutung hatten als im Westen.

\section{Die Pressburgische Gesellschaft}

Obwohl die Geschichte der Pressburgischen Gesellschaft gut dokumentiert ist, weiß man über die Entstehung und über derer Anfangszeiten nicht viel. Wie ich in meinem erwähnten Aufsatz formulierte: „Die [unter der Bedingungen des damaligen Königreichs Ungarn] relativ frühe Entstehung dieser Sozietät könnte als ein Modell der Wirkung der lebendigen Ideen der Aufklärung in Mittel-Osteuropa und auch als ein Beweis dafür gelten, dass das Streben des Bürgertums nach politischem und gesellschaftlichen Selbstbewusstsein im multikulturellen Raum von Pressburg vor dem Zeitalter des Nationalismus entstehen konnte. “4 Es gab Experimente und Bemühungen, eine Gelehrtengesellschaft früher und zu der gleichen Zeit zu stiften, aber allein Karl Gottlieb Windisch und sein Kreis hat es erreicht, einige Jahre lang als solche zu funktionieren, und nur eben wegen dieser dokumentierten Praxis kann man seinen Kreis „Gesellschaft“ nennen. Aber ihre Einzigartigkeit und die relativ späte Entdeckung der Archivalien der Gesellschaft (erst im 20. Jahrhundert), ihre Identität (Lutheraner, deutschsprachig) wurden in der Forschungsgeschichte ziemlich lange wichtiger genommen, als ihre Zielsetzungen. ${ }^{5}$

3 Hegedüs (Anm. 1), S. 55.

4 Ebd., S. 53.

5 Ebd. 
Wir besitzen heute drei verschiedene zeitgenössische Dokumente über die Gesellschaft. Das erste ist ein Entwurf der Gesetze oder Statuten der Gesellschaft jene Gesetze, die aber nicht angenommen wurden. Das 1936 von Fritz Valjavec veröffentlichte Manuskript muss sich bis heute in Pressburg befinden. ${ }^{6}$ Erst 1944 berichtet Béla Iványi über das neuentdeckte vollständige Gründungsdokument der Gesellschaft. Die von Iványi angeführten Hauptteile muss man mit der Liste der vorgetragenen Aufsätze ergänzen. Die Hauptteile sind: Kurz gefaßte Nachrichten von der Preßburgischen Gesellschaft der Freunde der Wissenschaften, in dem allgemein die Geschichte und die Ziele der Gesellschaft behandelt wurden. Den nächsten Teil bilden die Gesetze der Preßburgischen Gesellschaft der Freunde der Wissenschaften. Diese sind die endgültigen Gesetze, die sich in sehr wichtigen Punkten vom Entwurf unterscheiden. Dann kommt die Liste der Vorgelesenen Arbeiten. Sie besteht aus 84 Titeln. Im Verzeichnis deren Einverleibten Mitgliedern dieser Gesellschaft sind 10 Personen genannt. ${ }^{7}$ Darauf folgen manche Aufsätze, die man mit anderen, aus den Forschungen von Andrea Seidler und László Szelestei Nagy ergänzen kann. ${ }^{8}$ Nach Éva V. Windisch, die eine andere Version oder Kopie der genannten Dokumente gefunden und vorgestellt hat, wurden diese für eine geplante deutsche Publikation zusammengestellt. ${ }^{9}$

Aus den Dokumenten ist ersichtlich, dass die Pressburger Intellektuellen unter der Führung von Karl Gottlieb Windisch die Gesellschaft eigentlich zweimal gegründet haben. Zuerst verbanden sich Anfang 1752 einige Freunde und Liebhaber der Wissenschaften, den Beispielen so vieler Vorgänger nachzufolgen. (Selbst Windisch war schon lange Mitglied verschiedener Gelehrtengesellschaften, z. B. in

6 Fritz Valjavec: Die Preßburger Gesellschaft der Freunde der Wissenschaften (1761-1762). In: Ungarische Jahrbücher (1936), S. 265 f.

7 Béla Iványi: Die Pressburger Gesellschaft der Freunde der Wissenschaften. In: Südostforschungen 9-10 (1945-1944), S. 251.

8 Andrea Seidler: Gelehrte Gesellschaften in Ungarn und deren Verbindungen zum Zeitschriftenwesen im 18. Jahrhundert. In: Das achtzehnte Jahrhundert und Österreich. Jahrbuch der Gesellschaft zur Erforschung des achtzehnten Jahrhunderts 5 (1988), S. 41-53; László Szelestei N.: A pozsonyi Gesellschaft der Freunde der Wissenschaften [Die Pressburger Gesellschaft der Freunde der Wissenschaften]. In: Kolligátum: Tanulmányok a hetvenéves Bíró Ferenc tiszteletére. Hg. von Balázs Devescovi u.a. Budapest 2007, S. 404-411; László Szelestei N.: Tudományok és irodalom, latin és anyanyelv Windisch Károly Gottlieb pozsonyi tudós társaságában (1752-1762) [Wissenschaften und Literatur, Latein und Muttersprache in der Pressburger Gelehrtengesellschaft von K. G. Windisch]. In: Scientiarum miscellanea: Latin nyelvü tudományos irodalom Magyarországon a 15-18. században. Hg. von Péter Kasza u.a. Szeged 2017 (Convivia Neolatina Hungarica 2), S. 219-228.

9 Éva V. Windisch: Kovachich Márton György, a forráskutató [Márton György Kovachich, der Quellenforscher]. Budapest 1998, S. 50. 
Augsburg, und war auch gleichzeitig selbstverständlich Freimaurer.) Sie wollten mit ihren „wenigen Kräften“ „den Geschmack bessern“ und „den Witz [Ratio] schärfen“. Diese Entstehungsphase der Gesellschaft kann man noch nicht als öffentlich bezeichnen. Die Mitglieder wollten sich - nach dem Horazschen Vorbild „von dem bloß sinnlichen Treiben des Pöbels unterscheiden“ und „in vergnügter Stille und Ruhe“ die Zeit nützlich gebrauchen. ${ }^{10}$

Bei den meisten, insgesamt achtzehn Vorlesungen geht es um geschichtliche Themen, vor allem um die Geschichte des Vaterlandes. Die für die Ureltern der Ungarn gehaltenen Hunnen müssen eine wichtige Rolle gespielt haben, Windisch hat auch eine Ode über sie geschrieben. Literarische, sprachwissenschaftliche Themen wurden in dreizehn Vorträgen berührt. Darinnen vermischen sich poetische Werke und auch Abhandlungen. Philosophische, psychologische Themen sind ebenfalls zu finden, z. B. eine Abhandlung vom Nichts. Daneben kann man zahlreiche naturwissenschaftliche, astronomische, moralisierend-populäre Vorlesungstitel finden. Béla Iványi, der diese Dokumente entdeckt und erstmals vorgestellt hat, hat die Beobachtung gemacht: „Die Titel der Vorlesungen beweisen uns auch ganz genau, daß die Gesellschaft eine aufklärische Tendenz hatte. Lob der Unbeständigkeit oder Vom Vorurteile, dann: Die Ehre ein Vorurteil sind Titel, die vermuten lassen, daß man solche Vorträge auch in einer Freimaurerloge mit einstimmigen Beifall ruhig vorlesen könnte. “11

Das halte ich für eine sehr wichtige Beobachtung, die vielleicht die heutigen Gesellschaftsforschungen auch beeinflussen könnte: wie kann man heute die verschiedenen Gesellschaftstypen, unter anderen die Freimaurerei und gelehrten Gesellschaften voneinander unterscheiden, wenn derer vorgetragene Themen dafür keinen Grund geben, und wenn daneben, wie Markus Meumann formuliert: „,die] Aufsatzpraktiken in vielen Gesellschafts- bzw. Sozietätstypen des 18. Jahrhunderts einschließlich der Geheimbünde eine wesentliche Rolle für das gemeinschaftliche Tun [gespielt haben]?“12

Einen weiteren Beleg für diese These habe ich im von Andrea Seidler herausgegebenen Briefwechsel des Karl Gottlieb Windisch gefunden, aus eben den 1750er Jahren. Windisch korrespondiert mit Johann Daniel Herz aus Augsburg über die Publikation der Vorlesungen einer dortigen gelehrten Gesellschaft, in welcher beide Mitglieder waren. Windisch spricht in allen Briefen Herz als

10 Hegedüs (Anm. 1), S. 58.

11 Iványi (Anm. 7), S. 256.

12 Markus Meumann: Logenreden und Übungslogen. Zur Praxis des Sprechens und Schreibens über vorgegebene Themen in der Freimaurerei des 18. Jahrhunderts. In: Aufklärung [Themenheft: Aufsatzpraktiken im 18. Jahrhundert. Hg. von Markus Meumann u.a.] 28 (2017), S. 239-274, hier S. 239. 
„Allerliebster Bruder, Theuerster Freund!“ an. ${ }^{13}$ Damit möchte ich nun hervorheben, dass Freimaurer zu sein eine so starke Identität bildete, dass es auch die Beschäftigung mit wissenschaftlichen Themen beeinflussen konnte.

In der endgültigen Version der Statuten oder Gesetze der Pressburger Gesellschaft sind noch zwei wichtige Punkte $\mathrm{zu}$ finden, derer freimaurerischer Ursprung - meiner Meinung nach - unleugbar ist. Der zweite Punkt lautet: „Überhaupt sind die Mitglieder nicht nur an keine Zahl gebunden, sondern sollen auch ohne Ansehen der Religion, Stand, und Nation in die Gesellschaft aufgenommen werden.“ Im 7. Punkt ist Folgendes zu lesen: „Es ist keinem erlaubt in seinen Ausarbeitungen irgend etwas zu behaupten, oder einfliessen zu lassen, was wider die Religion, den Staat, die Ehrbarkeit, und Tugend laufet." ${ }^{14}$ Diese zwei Punkte sind leicht aus der Konstitution von James Anderson ableitbar.

Die Vermutung über den freimaurerischen Ursprung dieser Gesellschaft habe ich in meinem schon erwähnten Aufsatz folgendermaßen ausgedrückt: „Die zahlreichen Quellenausgaben westlicher Gesellschaften zeigen, dass sie allgemein sehr ordentlich dokumentiert wurden. [Ü]ber die Tätigkeit und Zielsetzungen der Pressburgischen Gesellschaft sind auch drei Dokumente erhalten. Diese drei wurden erst im 20. Jahrhundert entdeckt, und es scheint so, dass sie auch für die Zeitgenossen unbekannt waren. Das kann zwei Gründe haben: Entweder wurden die Arbeit und die Ergebnisse der Gesellschaft wegen einer nötigen Geheimhaltung nicht öffentlich dokumentiert und verbreitet, oder die Mitglieder haben einfach kein Publikum für ihre Zielsetzungen gehabt.“ - schrieb ich 2002. ${ }^{15}$ Heute kann ich eine ganz andere Deutung auch nicht ausschließen: Vielleicht funktionierte die Gesellschaft immer als eine Freimaurerloge, derer Name bis heute unbekannt ist. Die Vorlesungen über die Themen wurden in dieser Loge vorgetragen, aber Windisch, der Mitglied westlicher Gelehrtengesellschaften und Freimaurerlogen war, hat versucht, die Loge in eine öffentlich arbeitende Gelehrtengesellschaft umzuwandeln. ${ }^{16}$ Dafür kann man auch finanzielle Gründe finden. Gelehrtengesellschaften arbeiteten - wie erwähnt - immer in Hof- oder Machtzentren, und

13 Karl Gottlieb Windisch: Briefwechsel des Karl Gottlieb Windisch. Hg. von Andrea Seidler. Budapest 2008 (Magyarországi tudósok levelezése 5), S. 3.

14 Hegedüs (Anm. 1), S. 60.

15 Ebd., S. 50.

16 Eine ganz ähnliche Folgerung hat Markus Meumann aus der Tätigkeit von Ignaz Born gezogen: „Mann könnte daher vermuten, dass Born mit den von ihm initiierten Übungslogen letztlich nur das illuminatische, vom Ordensgründer Adam Weishaupt 1778 entworfene Programm des Ordens als einer »Weisheitsschule«, die in den unteren Graden als eine Art "gelehrte Gesellschaft« oder »Academie« geführt werden sollte, umgesetzt habe.” Meumann (Anm. 12), S. 243. 
wurden allgemein vom Staat oder von einer Stadt unterstützt, da die Ziele der gelehrten Mitglieder und der (unbedingt) aufgeklärten Herrscher nahezu gleich waren. Die Pressburger haben vielleicht nach westlichen Mustern versucht, eine Umwandlung durchzuführen, in einem Land, wo, wie ich schon gesagt habe, das gesellschaftliche Projekt der Aufklärung nie durchgeführt wurde.

\section{Gelehrte Gesellschaften und Wissenschaftstheorie}

Mit den bisherigen Ausführungen wollte ich einen Kontext aufzeigen, der ohne Zweifel hinter den wissenschaftstheoretischen Überlegungen der Gesellschaft stehen kann. Der damalige Geschichtsschreiber der Pressburger formuliert folgendermaßen: „Die schönen Wissenschaften überhaupt waren der Gegenstand ihrer Bemühungen. Die Geschichte, und in Sonderheit die Historie des Vaterlandes, die Altertümer, die Erde Beschreibung, die Natur Kunde, die Sittenlehre, Rede Kunst, und Dicht Kunst eröffneten ihnen ein weites, und fruchtbares Feld zu bearbeiten." ${ }^{17}$

Daraus folgt, dass der Autor des Textes die sogenannten „schönen Wissenschaften“ von allen anderen Zweigen der Wissenschaft abgrenzt, und darunter spezielle Forschungs- und Wissenschaftsfelder ordnet. Um diese Abgrenzung zu verstehen, braucht man eine klare Definition der „schönen Wissenschaften“, die ich dem Handbuch Europäische Literatur, Artikel Literatur von Stephan Matuschek entnehme: „Der engere Literatur-Begriff grenzt im 18. Jh. nicht (wie heute) den Bereich der sprachlichen Kunstwerke ein; es geht vielmehr darum, einen bestimmten Bereich der Wissenschaften und Künste $\mathrm{zu}$ definieren. Der LiteraturBegriff ist in dieser Entwicklung eng mit dem der belles lettres verbunden, auf Deutsch: dem der Schönen Wissenschaften, und er dient dazu, die wortsprachlich fundierten Wissenschaften von den mathematisch-naturwissenschaftlichen Disziplinen zu trennen. Dieser engere Literatur-Begriff fasst Dichtung, Geschichtsschreibung, Altertumskunde, die Philologien, Philosophie, Kunstkritik und alle rhetorischen Gattungen zusammen und grenzt sie gemeinsam von der Mathematik und den Naturwissenschaften, aber auch von der Rechtswissenschaft, der Medizin und der Theologie (den ehemals höheren Universitätsfakultäten) ab. “18 Für diese Abgrenzung habe ich in den letzten Jahren sprachtheoretische Gründe

17 Hegedüs (Anm. 1), S. 58.

18 Stephan Matuschek: Literatur. In: Handbuch Europäische Literatur: Begriffe - Konzepte Wirkung. Hg. von Heinz Thoma. Stuttgart-Weimar 2015, S. 335-343, hier S. 337. 
angegeben, da mich nicht die Abgrenzung der „schönen Wissenschaften“, sondern die Absonderung der Literatur im heutigen Sinne interessierte. ${ }^{19}$ Jetzt sehe ich ein, dass ich eine Stufe dieses Prozesses ignoriert habe. Matuschek aber hält diesen engeren Literatur-Begriff für so bedeutend, da er mit dessen Hilfe die Aufklärungsepoche der Literaturgeschichte am leichtesten definieren kann: „[...] die Parallelität von philologischer Wissenschaft, journalistischer Gebrauchsform, Dichtung und philosophischer Abhandlung ergibt die Einheit des engeren aufklärerischen Literatur-Begriffs. Die künstlerischen Formen [...] sind darin nur ein Ausdrucksregister unter anderen. Sie bilden keinen autonomen Bereich der Kunst, sondern stehen solidarisch im Verbund mit journalistisch-pragmatischen, wissenschaftlich argumentativen, rhetorisch zweckgerichteten Formen. ${ }^{20}$ Denken wir nach: es wäre zu schön unter dem Label „schöne Wissenschaften“ aufgrund des Aufklärungsprojekts eine Menge primär- oder sekundärliterarische Texte als Philologe zu interpretieren. Das würde bedeuten: wir haben heutzutage das Privileg Texte der Vergangenheit oder der Gegenwart 1. unabhängig von ihrem Kontext, oder 2. im beliebigen Kontext zu interpretieren. Wir könnten so Teilnehmer eines Sprachspiels sein, die damaligen aber nicht. Dabei bleibe ich aber skeptisch.

Im Briefwechsel von Windisch, adressiert an den erwähnten Herz, kann man die folgenden lesen: „Die Herausgabe einzelner Bögen [einer geplanten Zeitschrift der Augsburger gelehrten Gesellschaft], von so verschiedener Materie ist unnatürlich, folglich lächerlich, und wider die Regeln der Klugheit.“21 Es scheint so, dass er klar die Unterschiede der Kontexte einer Publikation und einer Gesellschaft fühlt, obwohl in beiden Fällen es um die Schönen Wissenschaften geht. Oder, wie er im nächsten Brief auseinandersetzte: „Nur ist mir der Titel Kunstschrift für die Beiträge der Gelehrten zu unnatürlich. Kunstschrift bedeutet im wahren Verstande eine künstliche Schrift; oder eine Anleitung, wie man schön und künstlich schreiben solle. [...] Abhandlungen und Gedichte einiger Mitglieder, wie ich schon oft erwähnte; oder Arbeiten einiger Mitglieder, würde in der Mittelstraße der beste, und vernünfigste Titel seyn!“22

19 Béla Hegedüs: A szimbolikus gondolkodás és az irodalom születése [Das symbolische Denken und die Geburt der Literatur]. In: Stephanus noster: Tanulmányok Bartók István 60. születésnapjára. Hg. von József Jankovics u.a. Budapest 2015, S. 383-393; Béla Hegedüs: Epistemologischer Hintergrund des Litterae-Literatur-Überganges im 18. Jahrhundert: Ein Versuch. In: Germanistische Studien 9. Hg. von Mihály Harsányi. Eger 2013. S. 49-57.

20 Matuschek (Anm. 18), S. 337.

21 Windisch (Anm. 13), S. 3.

22 Ebd., S. 5. 
Meiner Meinung nach folgt daraus, dass auch den Damaligen die verschiedenen Lesarten als verschiedene Interpretationsmöglichkeiten zur Verfügung standen. Damit leugne ich nicht ab, dass die ästhetisch beurteilbare Literatur noch Teil der sogenannten schönen Wissenschaften war und damit auch epistemologische Funktionen hatte, aber man muss bemerken, dass ihre Absonderung tief in der Aufklärungsepoche begonnen hatte, vielleicht mindestens im Falle von Karl Gottlieb Windisch. 